\title{
Berichtigungen
}

zu: O. C. Hilgenberg, Die Bruchstruktur der sialischen Erdkruste

Seite 29. Zeile 1: Moeara, an Stelle von: Moera

Seite 28, Zeile 28: $\mathrm{N} 60^{\circ} \mathrm{W}$, an Stelle von: $\mathrm{N} 60^{\circ} \mathrm{E}$

Seite 28, Zeile 30: $10^{\circ} \mathrm{N}$, an Stelle von $10^{\circ} \mathrm{S}$

Seite 28, letzte Zeile: $N 78^{\circ} \mathrm{E}$, an Stelle von: $\mathrm{N} 78 \circ \mathrm{W}$

Seite 43, Zeile 2: 1:2000 000, an Stelle von: 1:20000000

Seite 49. Zeile 29: Jelstrup- und Overbye-Fjellet, an Stelle von: Jelstrup-Overbye-Fjellet

Seite 89, Zeile 2: dem die betreffenden, an Stelle von: dem betreffenden

Seite 99, Zeile 11: Die, an Stelle von: Dei 



\section{DIE BRUCHSTRUKTUR}

\section{DER SIALISCHEN ERDKRUSTE}

VON

DR.-ING. OTT CHRISTOPH HILGENBERG

MIT 4 TAFELN UND 40 ABBILDUNGEN

$$
1949
$$

AKADEMIE-VERLAG BERLIN 
Copyright 1949 by Akademie-Verlag, Berlin Alle Rechte vorbehalten

Erschienen im Akademie-Verlag GmbH., Berlin NW 7, Schiffbauerdamm 19

Verlags-Lizenz-Nr. $156 \cdot 3592 / 48-3182 / 48$

Gedruckt vom Leipziger Druckhaus, Leipzig (M I15)

Bestell- und Verlags-Nr. 5022 